\title{
Dynamics of immigrants' health: \\ Evidence from Canada, 1994-95 to 2002-03. Summary Report
}

\section{Edward Ng}

Health Analysis and Measurement Group

Statistics Canada

Ottawa, Ontario, Canada

\section{Russell Wilkins}

Health Analysis and Measurement Group

Statistics Canada

Ottawa, Ontario, Canada

Department of Epidemiology and Community Medicine University of Ottawa

Ottawa, Ontario, Canada

François Gendron

Health Analysis and Measurement Group

Statistics Canada

Ottawa, Ontario, Canada

Jean-Marie Berthelot

Health Analysis and Measurement Group

Statistics Canada

Ottawa, Ontario, Canada

Department of Epidemiology and Biostatistics

McGill University

Montreal, Quebec, Canada 
Ng, Edward, Russell Wilkins, François Gendon

and Jean-Marie Berthelot

Recent immigrants from non-European countries were twice as likely as the Canadian-born to report deterioration in their health over an eight year period, according to a new study on the dynamics of the health of immigrants using longitudinal data ( $\mathrm{Ng}$ et al, 2005).

When immigrants arrive in Canada, they are generally in better health than the Canadian-born population. Potential immigrants are screened on medical and other health-related criteria before they are admitted to the country. There is also a degree of self-selection in the originating country, with applicants likely to be individuals with the stamina and motivation to undertake the rigours that immigration entails. An earlier study found that $97 \%$ of new immigrants rated their health as good, very good or excellent six months after their arrival, compared with $88 \%$ for the general population. However, as time passes, this so-called 'healthy immigrant effect' tends to diminish as their health status converges with that of the general population.

Most studies of immigrant health have been based on cross-sectional data, and so have not been able to assess the health impact on immigrants as they settle into the host country. This study followed initially healthy individuals across five waves of longitudinal data from the National Population Health Survey. The survey covered private households and institutional residents in all provinces, except on Indian reserves, Canadian Forces bases, and some remote areas. The first cycle of the survey (in 1994-95) interviewed 14, 117 Canadians aged 18 and above living in private households. These individuals were then followed over time. For people who rated their health as good, very good or excellent in 1994-95, changes in health status, health care utilization (frequent doctor contacts and hospitalization) and health-related behaviours (daily smoking, leisure activity and body mass index) over time were compared by European/non-European origin and duration of residence in Canada.

The discrete proportional hazards model used in this analysis accounted for differences in age, sex and socio-economic status, specifically, education levels and household income, as reported by respondents in 1994-95. These factors also tended to be significantly associated with deterioration in self-reported health status. For the estimates of variances and confidence intervals in all analyses, bootstrap weights were used to account for clustering and the unequal probabilities of selection into the survey.

The results showed that between 1994-95 and 2002-03, immigrants in general were more likely than the Canadian-born population to report a shift towards fair or poor health. Moreover, recent immigrants from non-European countries who arrived in Canada between 1984 and 1994 were twice as likely as Canadian-born to report deterioration in their health during the study period. 
Dynamics of Immigrants' Health:

Evidence from Canada 1994-95 to 2002-03, Summary Report

This might suggest that these recent immigrants from non-European countries, who were initially healthy in 1994-95, adopt some unhealthy lifestyles in the process of adapting to a new culture. Indeed, the study found that these immigrants were almost twice as likely as Canadian-born to have a $10 \%$ or more increase in their body mass index. This index is a measure to determine a person's weight relative to his or her height. An increase in this index reflects a weight gain, which might be harmful for one's health. As for daily smoking, no corresponding evidence was found as non-European immigrants overall were less likely than the Canadian-born to become daily smokers. The fact that recent non-European immigrants were at higher risk of experiencing deterioration in their health is mirrored in their becoming frequent visitors to the doctor (at least six consultations a year).

Immigrants overall make up an increasing share of the total population (18.4\% in 2001) and without sufficient immigration to compensate for belowreplacement fertility, the Canadian population could start to decline in about 30 years. Moreover, immigrants from non-European countries constitute an increasingly important segment of Canadian society, as about three-quarters of recent immigrants come from non-European nations. With longitudinal data, it is possible to document the decline in immigrants' health and broadly identify the group most affected: those of non-European origin. A better understanding of the dynamics behind the changes in immigrants' health could inform public policy about the potential risks that confront this increasingly important component of Canadian society.

\section{Reference:}

Ng, Edward, Russell Wilkins, François Gendron, and Jean-Marie Berthelot. 2005. "Dynamics of immigrants' health in Canada: evidence from the National Population Health Survey." Healthy today, healthy tomorrow? Findings from the National Population Health Survey. Ottawa: Statistics Canada catalogue 82618; 2:1-11. Available free at: http://www.statcan.ca/english/research/82-618MIE/82-618-MIE2005002.htm

Address for correspondence: Edward Ng, Health Analysis and Measurement Group, Statistics Canada, RHC-24R, Ottawa ON K1A 0T6;

edward.ng@statcan.ca. 